\title{
FACTORS AFFECTING RESULT IN CHINESE PROFICIENCY TEST (HSK) LEVEL 6: READING SECTION AND PREPARATION STRATEGIES
}

\author{
影响HSK6级阅读的因素及应对策略
}

\author{
Sri Haryanti \\ Sichuan Normal University \\ Rongyuan 1405 No 5 Jing'an Road, Jinjiang District, Chengdu, Sichuan, PR China 610066. \\ thincele@yahoo.com
}

\begin{abstract}
Chinese Proficiency Test (HSK) is an internationally standardized exam which tests and rates Chinese language proficiency. The highest level in this test is level 6. The writing part of the test consists of 3 (three) parts, namely, (1) listening, (2) reading, (3) writing. Furthermore, the reading part is made of 4 components. Level 6 of this test implies a high degree of difficulty. This paper specifically looked on how to prepare effectively for participants to be able to work on the reading part in order to achieve best result. This article used the methods of literature review and observational study as well as field research and would also incorporate the author's personal experience in taking the test into recommending strategies for doing the reading part in a level 6 HSK test. Finally, research suggested several techniques and tips that might assist participants in achieving maximum scores in handling the reading part of level 6 HSK test.
\end{abstract}

Keywords: Chinese Proficiency Test (HSK), reading difficulty

摘要

新汉语水平考试是国际汉语能力标准化考试，新汉语水平考试的最高等级是6级，考试内容分为四个部 分, 其中阅读是其中一个重要组成部分。对于考生来说, 新汉语水平考试6级的阅读部分难度较高, 考生新汉 语水平6级考试阅读部分做的结果的好坏，直接关系着其是否能顺利通过6级考试。这篇论文主要以文献研究法 和调查法两种方式, 结合前人的研究成果和自己新汉语水平考试的经验实践, 提出了解决新汉语水平考试6级 阅读问题的一些技巧和应对策略，希望能给要考汉语6级的朋友一些借鉴。

关键词：新汉语水平考试6级，阅读，难度，问题和技巧 
前言

新汉语水平考试是一项国际汉语能力标准化考 试。在新汉语水平考试6级考试分三个部分、听力、 阅读和书写。，按写着的经验考生对阅读部分觉得难 度较高, 所以这本论文只是写关于阅读部分。通过跟 其他留学生的研究, 大多留学生会觉得阅读部分是最 难的。怎样面对新汉语水平考试阅读部分, 还有考试 如何备考新汉语水平考试是这篇论文写得目的。通过 这篇论文希望能帮助考生如何面对新汉语水平考试特 别是阅读部分。

\section{新汉语水平考试6级阅读部分介绍}

新汉语水平考试是一项国际汉语能力标准化考 试, 重点考查汉语非第一语言的考生在生活、学习和 工作中运用汉语进行交际的能力。新汉语水平考试有 6级, 新汉语水平考试6级 (HSK6级) 是其中之一。 新汉语水平考试6级考查考生的汉语应用能力, 通过 HSK6级的考生可以轻松地理解听到读到的汉语信息, 以口头或书面的形式用汉语流利地表达自己的见解。

新汉语水平考试6级主要面向掌握5000及5000 以上常用词语的考生。考查内容包括听力部分, 考题 共有 50 题、阅读部分, 考题共有 50 题和书写部分, 书 写共有 1 题, 全部考试约 140 分钟 (含考生填写个人信 息时间5分钟）。

新汉语水平考试6级阅读分四个部分：第一部 分, 共 10 题。每题提供 4 个句子, 要求考生选出有语 病的一句; 第二部分, 共 10 题。每题提供一小段文 字, 其中有 3 到 5 个空格, 考生要结合语境, 从 4 个选 项中选出最恰当的答案; 第三部分, 共 10 题。提供 两篇文字, 每篇文字有5个空格, 考生要结合语境, 从提供的 5 个句子选项中选出答案; 第四部分, 共 20 题。提供若干篇文字, 每篇文字带几个问题, 考生要 从 4 个选项中选出答案。阅读部分共有 50 题, 考试时 间有45分钟。如果看考查题和时间举行考生要掌握好 两个部分, 就是考题和时间, 每题在 1 分钟左右必须 做好。如果考虑到每个部分的难度, 做好每个题都还 要看具体情况。

\section{新汉语水平考试6级阅读难度}

在新汉语水平考试6级阅读部分每个部分难度 都不同。在第一部分, 要求考生选出有语病的一句。 对考生来说这个部分难度较高, 所以有的考生选择最 后才做这个部分。在这个部分让考生选择有语病的句 子加上时间很紧难度相当高。病句难度有一些原因 如: 每个句子长短不同、有一些不太熟悉的词汇、读 的速度还有考生不知道到底哪一个原因会影响那个句 子成为病句。从这些原因来看我们可以看如果一个题 句子较长的话, 对考生来说会带来麻烦, 就是看长句 子都会让考生产生紧张心理, 还有如果读的速度不快 会影响到选择答案。有时候考生读的速度挺快可是碰 到了不熟悉的词汇也会影响到他们的答案。最糟糕就 是如果每个句子都已经读完但还是不知道到底是哪一 句有病句。这样就会花很多时间去考虑。

在第二部分，要求考生从 4 个选项中选择出最 恰当的答案, 每题提供一小段文字, 其中有 3 到 5 个空 格, 考生要结合语境。考生要理解词汇的用法、单位 和词汇的近义词。有时候考生碰到在四个选项中有不 熟悉或不懂怎么用的词汇, 这样也会产生紧张而变成 随便选择答案。也有时候只要看前面的答案而没有注 意到最后的答案就直接选择, 这样也会出现错误。

第三部分，提供两篇文字，每篇文字有 5 个空 格, 考生要结合语境, 从提供的 5 个句子选项中选出
答案。在这个部分一般的文字有点儿长, 还有内容 有各种各类的类型, 如文化、科学、技术等。考生一 看比较长的文字就会感到很紧张, 还有文字的内容也 不熟悉, 读的速度不那么快, 都会影响考生选择的答 案。也有考生把所有的文字读完才回答, 这样会浪费 很多时间。

第四部分，提供若干篇文字，每篇文字带几个 问题, 考生要从 4 个选项中选出答案。每篇文字长短 和难度不同, 如果碰到较难的文章考生就紧张而且一 直想那篇文章这样会浪费时间。第四部分共有 20 题, 大概有 4 到 5 篇文字。每篇文字内容不同, 有关于生活 方面、科学方面或艺术方面等等。每个考生的兴趣也 不同, 所以碰到比较熟悉的文章考生很快就会回答, 而碰到不熟悉的文章考生会花较长的时间。不只是内 容会影响考生做好第四部分还有另外的如: 词汇量, 每篇文字大概有 400 到 500 个字而不是所有的词汇考生 都会读, 所以如果碰到不熟悉的词汇会影响考生的答 案; 读的速度, 有的考生读文字的时候速度有点慢, 考生无法抓紧时间这样会产生紧张而会随便选择答 案。

\section{结语}

新汉语水平考试是一项国际汉语能力标准化考 试, 重点考查汉语非第一语言的考生在生活、学习和 工作中运用汉语进行交际的能力。新汉语水平考试6 级是新汉语水平考试最高的考试部分。阅读是其中一 个考查部分。备考新汉语水平考试不是一两个月准备 的事, 而是贯穿考生学习汉语的全过程。新汉语水平 考试6级要求是考生已经掌握好 5000 个字以上词汇, 这个词汇不是要天天背的词而是通过考生平常学习过 程掌握到的词。

考生在报名后才开始准备考试会出现很多问 题, 就是因为考生的备考不够, 有时候他们也只是注 意被生词而已和不懂如何用那个词。这会影响考生对 阅读考试的问题, 还有如果在阅读速度不够快在考试 的时候也会影响考生的信心。

新汉语水平考试6级阅读部分, 需要考生对语法 理解和词汇量的掌握上要特别强。考生要多看报纸、 小说、杂志等, 了解很多文章的内容, 这样会增加考 生的词汇量还有培养提高阅读速度。在一般汉语课堂 特别是语法课上, 老师们也要强调语法理解、词语使 用等, 这样会帮助将来学生参加汉语水平考试。最重 要的是备考时, 考生要多做新汉语水平考试模拟考 试，每次都要严格按照考试时间举行模拟测试。培养 这样的习惯可以帮助考生减少考试时的紧张心理。每 个部分的时间要按照考生自己的能力来安排, 哪一个 部分较强的话时间的安排可以少一点。还有语法老师 要多鼓励学生理解语法和近义词的用法。还有最重要 的就是考生自己的心理要调整好, 这样可以帮助其轻 松应考。

\section{参考文献}

陈香. (2012). 《新HSK考试辅导教程6级》, 北京: 高等 教育出版社。

College of International Education. (2011, 2 November). 新 HSK六级介绍. Accessed from http://cie.upc.edu.cn/ cie/news_read.aspx?Eid=\%20118

李珠花. (2012). 《新汉语水平考试六级阅读第一部分病 句试题统计与分析》, [硕士学位论文]. 武汉: 华中科技大学。 